\title{
Um Estudo sobre Denúncias de Violência Registradas no Disque 100 -
}

\section{Pessoas com Deficiência}

\author{
Márcia de Lima* \\ Universidade Federal de São Carlos - UFSCar, São Carlos, SP, Brasil \\ ORCID: https://orcid.org/0000-0002-8956-8369 \\ Sabrina Mazo D'Affonseca** \\ Universidade Federal de São Carlos - UFSCar, São Carlos, SP, Brasil \\ ORCID: https://orcid.org/0000-0001-9103-0616
}

\section{RESUMO}

As pessoas com deficiência, quando comparadas a seus pares sem deficiência, são mais vulneráveis a sofrerem violência. $O$ presente trabalho tem por objetivo analisar os dados relativos às denúncias de violência contra pessoas com deficiência disponibilizados pelo Disque Direitos Humanos - Disque 100. Utilizou-se como fonte de dados o Disque 100 Balanço Anual 2017 - Pessoas com Deficiência, sendo analisados os dados referentes à agressão (frequência, tipo de violação e local); às características da vítima (deficiência, etnia, idade e sexo) e dos agressores (relação com a vítima, idade, sexo e etnia); quem foi o denunciante. Verificou-se um número considerável de denúncias entre os anos de 2011 e 2017 $(M=8800, d p=2879)$, sendo que a maior parte dos casos $(35,8 \%)$ foi de negligência (abandono, alimentação, amparo, higiene, medicamentos/assistência à saúde). As violações ocorreram no espaço privado do lar $(85,6 \%)$ e as vítimas eram em sua maioria mulheres $(52,8 \%)$, pardas $(29,7 \%)$, com idade entre 18 a 50 anos $(76,9 \%)$ e deficiência intelectual $(65,8 \%)$. A maioria dos perpetradores era familiares $(57,1 \%)$, homens $(57,6 \%)$, com idade entre 18 a 50 anos $(39,6 \%)$. Tal quadro sinaliza a necessidade de ações para a prevenção da violência cometidas contra pessoas com deficiência.

Palavras-chave: violência, deficiência, prevenção.

\section{A Study on Violence Reports Registered in Disque 100 - People with}

\section{Disabilities}

\begin{abstract}
People with disabilities are more vulnerable to violence when compared to their non-disabled peers. The purpose of this study was to analyze report data of violence against people with disabilities available by Human Rights Hotline (Disque 100). Data source was the Human Rights Hotline's (Disque 100) Annual Balance of 2017- People with Disabilities, and the data analyzed regarding type of aggression (frequency, type of violation and location);
\end{abstract}


characteristics of the victim (disability, ethnicity, age and sex) and aggressors (relationship to victim, age, sex and ethnicity); and who was the whistleblower. There was a considerable number of complaints between 2011 and $2017(\mathrm{M}=8800, \mathrm{SD}=2879)$, with the majority of cases $(35.8 \%)$ being negligence (abandonment, food, shelter, hygiene, medicines / health care). Violations occurred mostly at home $(85.6 \%)$ and the victims were mostly women (52.8\%), brown $(29.7 \%)$, aged between 18 and 50 years $(76.9 \%)$, and presented intellectual disability $(65.8 \%)$. The majority of perpetrators were family members $(57.1 \%)$, men $(57.6 \%)$, aged 18-50 years $(39.6 \%)$. Such a framework points the necessity of actions to prevent violence against people with disabilities.

Keywords: violence, disability, prevention.

\section{Un Estudio sobre Denuncia de Violencia Registradas en la Disque 100 -}

\section{Personas con Discapacidad}

\section{RESUMEN}

Las personas con discapacidad, en comparación con sus parejas sin discapacidad, son más vulnerables a sufrir violencia. El presente trabajo tiene por objetivo analizar los datos relativos a las denuncias de violencia contra personas con discapacidad disponibles por la Línea Directa de Derechos Humanos (Disque 100). Se utilizó como fuente de datos la Línea Directa de Derechos Humanos (Disque 100)- Balance Anual 2017 - Personas con Discapacidad, siendo analizados los datos relativos a la agresión (frecuencia, tipo de violación y lugar); a las características de la víctima (discapacidad, etnia, edad y sexo) y de los agresores (relación con la víctima, edad, sexo y etnia); y quién fue el denunciante. Se observó un número considerable de denuncias entre los años 2011 y $2017(\mathrm{M}=8800, \mathrm{DE}=2879)$, siendo que la mayoría de los casos $(35,8 \%)$ fueron de negligencia (abandono, alimentación, amparo, higiene, medicamentos/asistencia sanitaria). Las violaciones ocurrieron en el espacio privado del hogar $(85,6 \%)$ y las víctimas eran en su mayoría mujeres $(52,8 \%)$, pardas $(29,7 \%)$, con edad entre 18 a 50 años $(76,9 \%)$, y con discapacidad intelectual $(65,8 \%)$. La mayoría de los perpetradores eran familiares $(57,1 \%)$, hombres $(57,6 \%)$, con edad entre 18 a 50 años $(39,6 \%)$. Este cuadro señala la necesidad de acciones para la prevención de la violencia contra las personas con discapacidad.

Palabras clave: violencia, discapacidad, prevención.

Ao longo dos últimos anos, tem sido cada vez mais comum o reconhecimento de que as pessoas com deficiência são vulneráveis a sofrerem violências de diferentes tipos (Cavalcante et al., 2009; Ferreira, 2008; Moreira et al., 2014; Williams, 2003). Isso demanda a necessidade de se compreender esse fenômeno a fim de que ações preventivas possam ser propostas e realizadas. Apesar do alerta sobre a ocorrência de tais atos contra pessoas com 
deficiência, ainda são incipientes os dados relativos à prevalência, seja por registros inadequados, seja por dificuldade de acesso dessa população aos serviços.

Conceituar um fenômeno complexo como a violência requer considerar os diversos contextos e cenários em que este fenômeno pode ocorrer, além dos diferentes atores, considerando que ele está presente em todos os estratos sociais, independentemente de classe, renda ou nível de instrução, atingindo populações de diferentes religiões e culturas, com diferenciais baseados em gênero, idade e etnia (Cruz, Silva, \& Alves, 2007; Silva, Brito, Araújo, \& Abath, 2013). Estudos sobre a violência demonstram que uma grande e significativa gama de atos violentos ocorre dentro do ambiente doméstico (Day et al., 2003), sendo que as vítimas mais frequentes são mulheres, idosos, crianças e adolescentes. Segundo Barros, Deslandes e Bastos (2016), a violência que ocorre no seio familiar é prevalente, gera consequências físicas e mentais e tem sido reconhecida como problema de saúde pública. Pessoas que necessitam de cuidados complexos e contínuos encontram-se ainda mais vulneráveis às diversas formas de maus-tratos (físicos, psicológicos, negligência e abuso sexual).

Ao lado de faixa etária, gênero e situação socioeconômica, a deficiência está entre os diferentes fatores que podem aumentar a exposição da pessoa a atos de violência. Segundo Moreira et al. (2014), dados oficiais apontam que crianças e adolescentes com deficiências estão mais propensos a terem seus direitos violados. Além disso, a pessoa com deficiência constitui uma vítima fácil seja pela dificuldade em se comunicar, de identificar sinais de um possível abuso, de identificar o agressor e de evitar a agressão (Cavalcante et al., 2009; Ferreira, 2008).

A violência e a deficiência associam-se a fatores de risco, principalmente àqueles que estão relacionados à pobreza, moradia precária ou falta de moradia, ao isolamento social, às questões de gênero, às doenças físicas e mentais associadas à deficiência. De acordo com Williams (2003), o indivíduo com qualquer tipo de deficiência encontra-se em uma posição de grande vulnerabilidade em relação às pessoas sem deficiências, sendo marcante a assimetria das relações de poder na interação entre ambos. Outro fator apontado como condição para a violência contra pessoas com deficiência é a sua invisibilidade na malha social, que se caracteriza principalmente pela sua ausência física real da rede de relações. Segundo Ferreira (2008), o sujeito de direito é obscurecido pela condição de deficiência que possui, o que constitui uma barreira à luta pelos seus direitos.

A deficiência obscurece a pessoa-sujeito de direito que possui uma deficiência, independentemente de suas características. A condição imposta pela deficiência, então, 
destitui da pessoa sua essência como ser humano, pois suas outras dimensões humanas passam a não importar mais, uma vez que quando se constata (ou se pressupõe) a deficiência, esta condição generaliza-se rapidamente sobre a pessoa e toma seu lugar. Assim, a história e o currículo da pessoa com deficiência são descartados e, com eles, são ignorados e negligenciados seus direitos à voz, à participação e ao desenvolvimento pleno tornando-a cada vez mais vulnerável a toda a sorte de violência e discriminação, mesmo na vida adulta (Ferreira, 2008, p. 128).

A violência contra a pessoa com deficiência pode atingir todo o leque de direitos fundamentais, principalmente a educação e a saúde física e psicológica. O Estado está, portanto, obrigado a prevenir e a enfrentar a violência e a vulnerabilidade dos cidadãos, as quais são mais agravadas quando consideradas as pessoas com deficiência, sobretudo em vista do estigma social por elas enfrentado. A Convenção da ONU concernente aos Direitos das Pessoas com Deficiência impõe ao Estado e à sociedade tratarem da prevenção contra a exploração, a violência e o abuso de pessoas, tanto dentro como fora do lar (Pereira, Lopes, \& Gomes, 2015).

Segundo Pereira et al., (2015), as pessoas com deficiência têm mais dificuldades em acessarem serviços e em obterem intervenção da polícia, proteção jurídica ou cuidados preventivos quando necessário, seja por problemas de locomoção ou de comunicação da pessoa, seja por despreparo e concepções deturpadas em relação à condição de deficiência. Todavia, se a violação não é reconhecida nem denunciada, a invisibilidade se perpetua e, frequentemente, mesmo quando os casos são identificados, há dificuldades por parte das instituições em reconhecerem os direitos das pessoas com deficiência e as especificidades de suas violações (Coordenadoria Nacional para Integração da Pessoa Portadora de Deficiência, 2008). Para Williams (2003), dados internacionais reforçam a necessidade de um olhar específico para essa população, que tem 1,5 vezes mais chances de ser vítima de abuso sexual e 4 a 10 vezes maior probabilidade de ter vivenciado maus tratos quando criança.

A Constituição Nacional Brasileira de 1988 é um marco na luta pelo reconhecimento e proteção dos grupos vulneráveis, dentre eles as crianças e adolescentes e a pessoa com deficiência (Montibeller, 2012). A sua promulgação, propiciou a ampliação e a institucionalização de serviços de utilidade pública que se disponibilizam a receber denúncias de violações de direitos fundamentais de grupos vulneráveis, como é o caso das pessoas com deficiência. Nos anos de 1990, havia um movimento crescente em relação aos direitos das crianças e adolescentes, impulsionado tanto pela nova Constituição quanto pelo estabelecimento do Estatuto da Criança e do Adolescente (ECA). Esse movimento fez com 
que em 1997 fosse criado o Disque Denúncia - Disque 100, por organizações não governamentais (ONGs) que atuavam na promoção dos direitos das crianças e adolescentes (Ministério dos Direitos Humanos, 2018).

O Disque Direitos Humanos - Disque 100 tem abrangência nacional (Messeder, Boaventura, \& Oliveira Júnior, 2017) e consiste em um canal de comunicação entre a sociedade civil e o poder público para que se possa fazer denúncias sobre violações de direitos humanos. Em 2003 esse serviço passou a se chamar Disque Direitos Humanos - Disque 100, e ficou a cargo da Secretaria de Direitos Humanos, vinculada à Presidência da República e criada naquele mesmo ano, mas ainda era voltado somente para o público infanto-juvenil (Ministério Dos Direitos Humanos, 2018). Ao mesmo tempo em que ocorriam movimentos em defesa dos direitos do público infanto-juvenil, movimentos relacionados às pessoas com deficiência também aconteciam. Questões referentes às pessoas com deficiência eram conduzidas na esfera dos direitos humanos desde 1995, quando passou a existir, na estrutura do governo federal, a Secretaria Nacional de Cidadania do Ministério da Justiça (Maior, 2008).

No ano de 2009, o Brasil ratificou a Convenção dos Direitos das Pessoas com Deficiência, aprovada pela Assembleia Geral das Nações Unidas em 2006, através da resolução A/61/611, a qual, reconhecendo que mulheres e meninas com deficiência têm risco maior de sofrer violência, tanto no lar quanto fora dele, em seu artigo 16 descreve ações e passou a estabelecer metas para a garantia dos direitos das pessoas com deficiência (Brasil, 2012).

\section{Artigo 16}

Prevenção contra a exploração, a violência e o abuso

1. Os Estados Partes tomarão todas as medidas apropriadas de natureza legislativa, administrativa, social, educacional e outras para proteger as pessoas com deficiência, tanto dentro como fora do lar, contra todas as formas de exploração, violência e abuso, incluindo aspectos relacionados a gênero.

2. Os Estados Partes também tomarão todas as medidas apropriadas para prevenir todas as formas de exploração, violência e abuso, assegurando, entre outras coisas, formas apropriadas de atendimento e apoio que levem em conta o gênero e a idade das pessoas com deficiência e de seus familiares e atendentes, inclusive mediante a provisão de informação e educação sobre a maneira de evitar, reconhecer e denunciar 
casos de exploração, violência e abuso. Os Estados Partes assegurarão que os serviços de proteção levem em conta a idade, o gênero e a deficiência das pessoas.

3. A fim de prevenir a ocorrência de quaisquer formas de exploração, violência e abuso, os Estados Partes assegurarão que todos os programas e instalações destinados a atender pessoas com deficiência sejam efetivamente monitorados por autoridades independentes.

4. Os Estados Partes tomarão todas as medidas apropriadas para promover a recuperação física, cognitiva e psicológica, inclusive mediante a provisão de serviços de proteção, a reabilitação e a reinserção social de pessoas com deficiência que forem vítimas de qualquer forma de exploração, violência ou abuso. Tais recuperação e reinserção ocorrerão em ambientes que promovam a saúde, o bem-estar, o autorespeito, a dignidade e a autonomia da pessoa e levem em consideração as necessidades de gênero e idade.

5. Os Estados Partes adotarão leis e políticas efetivas, inclusive legislação e políticas voltadas para mulheres e crianças, a fim de assegurar que os casos de exploração, violência e abuso contra pessoas com deficiência sejam identificados, investigados e, caso necessário, julgados. (Brasil, 2012, p. 40-41)

Alguns anos depois, em 2011, o grupo das pessoas com deficiência passou a fazer parte do Disque 100. Ou seja, embora originalmente desenhado como uma ação estratégica para atender às denúncias de violação contra a população infanto-juvenil, posteriormente passou a atender também as denúncias contra outros grupos considerados como vulneráveis, entre eles, as pessoas com deficiência (Ministério Dos Direitos Humanos, 2018). As denúncias recebidas são analisadas e encaminhadas aos órgãos de proteção, defesa e responsabilização em direitos humanos, no prazo máximo de 24 horas, respeitando a competência e as atribuições específicas, porém priorizando qual órgão intervirá de forma imediata no rompimento do ciclo de violência e proteção da vítima (Ministério Dos Direitos Humanos, 2018).

Em estudo procurou conhecer os procedimentos e percepções de profissionais que atuam nos órgãos do Sistema de Garantia de Direitos (SGD) quanto à notificação da violência envolvendo crianças e adolescentes, realizado por Faraj et al (2016), os resultados apontaram um aumento de casos sendo notificados pela ferramenta do Disque 100. Segundo o estudo, nas falas dos participantes pode-se identificar que o Disque 100 tem sido uma ferramenta que auxilia nas notificações de violência, sendo visto pelos profissionais como uma forma mais 
fácil de fazer a denúncia, por ser uma ligação gratuita e sem a necessidade de identificação do denunciante.

Devido à importância desse mecanismo de proteção dos direitos humanos no Brasil, têm sido realizadas pesquisas que têm como fonte de dados as denúncias registradas no serviço Disque 100. Alguns focam em analisar a eficácia do serviço (Tavares, Louredo, \& Prado, 2013; Bernardes \& Moreira, 2013), outras em divulgar e analisar dados referentes às denúncias de violações de direitos, principalmente as que envolvem crianças e adolescentes (Andrade, 2012, 2017), idosos (Minayo, 2014), e o público LGBT (Messeder, Boaventura, \& Oliveira Júnior, 2017; Costa, 2016).

Porém, analisando o tempo de existência do serviço Disque 100 prestado pelo Estado para atender a população considerada vulnerável, fica evidente o desinteresse em entendê-lo no campo científico, uma vez que são escassos os estudos produzidos sobre o tema, e quando encontramos vincula-se principalmente ao grupo infanto-juvenil e à população LGBT. Em relação à população com deficiência, são encontrados apenas documentos institucionais, o que revela que a violência contra pessoas com deficiência no Brasil ainda é pouco estudada. Considerando que para prevenir a violência contra essa população o primeiro passo consiste em identificar a existência da mesma, assim como levantar características relacionadas ao perfil da vítima/agressor, locais de maior vulnerabilidade e tipos de violência comumente praticados, o presente trabalho tem por objetivo analisar os dados disponibilizados pelo Disque Direitos Humanos - Disque 100, de modo a caracterizar a violência contra pessoas com deficiência no Brasil.

\section{Método}

\section{Procedimentos}

\section{Coleta de dados}

Os dados foram levantados a partir das denúncias realizadas no Disque Direitos Humanos - Disque 100. O Ministério dos Direitos Humanos disponibiliza publicamente, em seu endereço na internet, um link para download dos dados obtidos pelo órgão ${ }^{1}$. Na página "Balanço Disque 100" 2 aparecem hiperlinks de acesso aos dados separados pelo tipo de população (criança e adolescentes, pessoa idosa, população em situação de rua, pessoa com deficiência, discriminação religiosa, pessoas em restrição de liberdade, igualdade racial, 
LGBT e outros). Na presente pesquisa, foram analisados os dados relativos ao "Balanço Geral - Anual 2011 a 2017- Pessoas com deficiência”. Os dados são disponibilizados pelo site em uma planilha Excel, organizados por ano, meses e unidades de federação para diferentes categorias (tipo de violação, perfil das vítimas, perfil dos suspeitos, relação suspeito x vítima, relação demandante x vítima, e local da violação), que são apresentadas em abas separadas no arquivo. Foram considerados registros feitos a partir de 2011, ano em que foram incorporados ao Disque 100 grupos com vulnerabilidade acrescida, entre eles, o grupo das pessoas com deficiência.

\section{Análise de Dados}

Os dados de domínio público disponibilizados pelo Disque 100 - Balanço Geral 2017 foram acessados no portal do Ministério dos Direitos Humanos, conforme descrito anteriormente, e analisados em sua totalidade. Após a análise total de todos os itens informados nas denúncias, foram selecionados os dados referentes ao número de denúncias, perfil das vítimas, perfil dos suspeitos, vínculos entre suspeitos e vítimas, denunciantes, vínculos entre denunciantes e vítimas e local da agressão, considerando-se o número total de denúncias, sem o recorte por meses e/ou unidade da federação. Os dados selecionados foram, então, organizados em uma nova planilha de Excel elaborada para a presente pesquisa. Todos os dados obtidos foram analisados a partir de uma estatística descritiva, calculando-se a frequência das variáveis.

\section{Resultados}

A Figura 1 apresenta o número total de denúncias realizadas no Brasil de 2011, ano que se incluiu o campo específico para identificar se a vítima tinha alguma deficiência, a 2017. De acordo com os dados da Figura 1, que representa o número total de denúncias no Brasil e por regiões, nota-se que entre os anos de 2011 a 2013 há uma reta ascendente, indicando um aumento do número de denúncias realizadas. Entre 2013 e 2014 há uma queda no número de casos, os quais se mantêm mais ou menos estáveis nos outros anos, com nova elevação em 2017, sendo um número pouco maior que o observado em 2013. Em relação às regiões brasileiras, verifica-se que as regiões Sudeste e Nordeste se destacam em números totais de caso. No entanto, é necessário ponderar o número de casos denunciados com o número de pessoas com deficiência nas diferentes regiões do Brasil. 


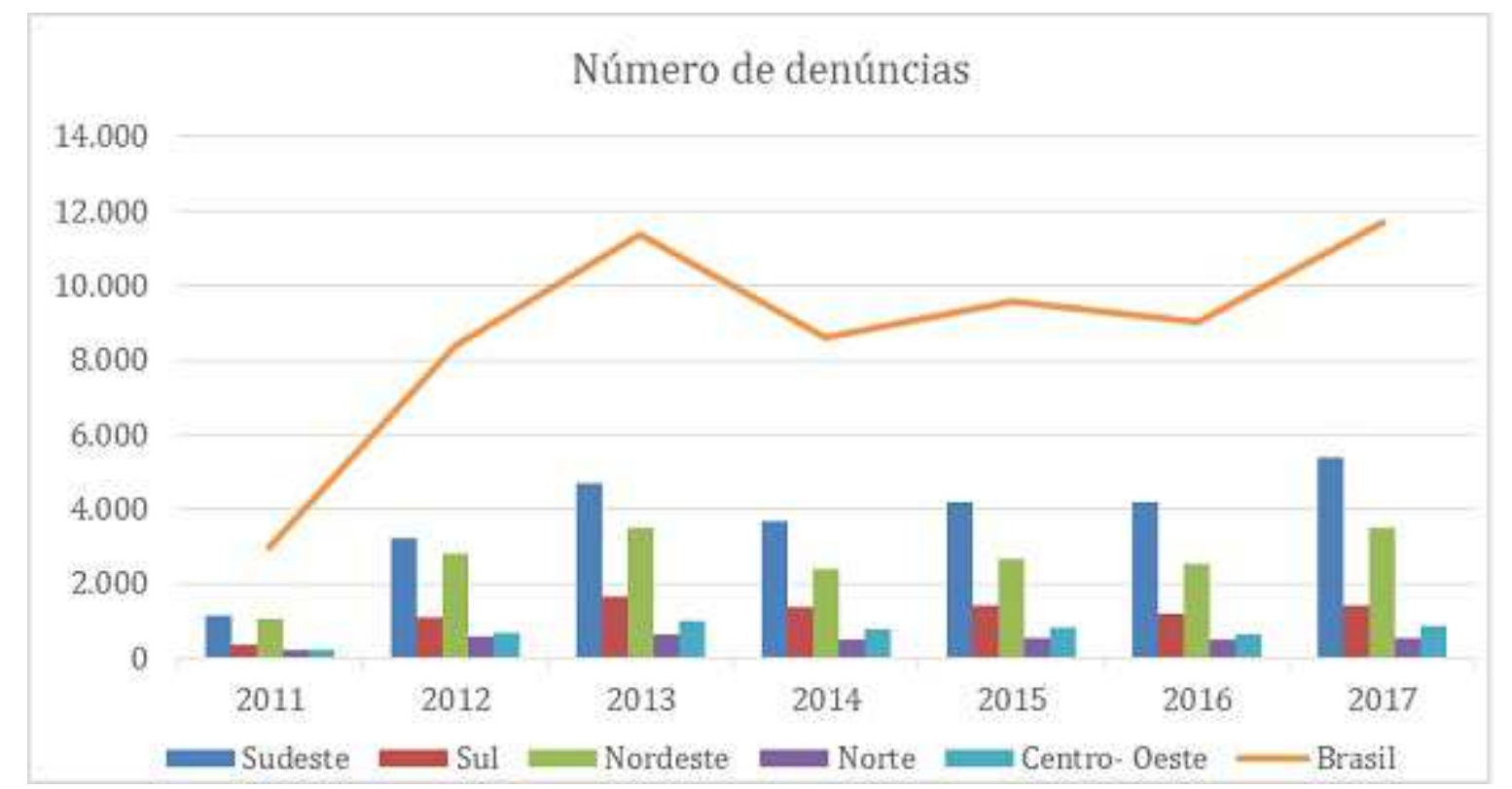

Figura 1. Número de denúncias de violações contra pessoas com deficiência no Brasil no período de 2011 a 2107.

De acordo com os dados da Figura 1, que representa o número total de denúncias no Brasil e por regiões, nota-se que entre os anos de 2011 a 2013 há uma reta ascendente, indicando um aumento do número de denúncias realizadas. Entre 2013 e 2014 há uma queda no número de casos, os quais se mantêm mais ou menos estáveis nos outros anos, com nova elevação em 2017, sendo um número pouco maior que o observado em 2013. Em relação às regiões brasileiras, verifica-se que as regiões Sudeste e Nordeste se destacam em números totais de caso. No entanto, é necessário ponderar o número de casos denunciados com o número de pessoas com deficiência nas diferentes regiões do Brasil.

\section{Caracterização da Violência}

A violência contra a pessoa com deficiência pode ocorrer de diferentes formas, referindo-se principalmente às ações e omissões que contrariam os direitos humanos. Segundo Abramovay (2002), a violência pode ser direta (física, sexual e negligência), indireta (ameaças, psicológica), econômica, e moral ou simbólica (discriminação). A Figura 2 apresenta os dados referentes aos tipos de violações mais denunciadas no período de 2011 a 2017. A maior parcela de casos denunciados no período foi relativa à negligência $(35,8 \%)$, seguida da violência psicológica $(25,5 \%)$, física $(17,7 \%)$ e do abuso financeiro $(13,3 \%)$. Outros tipos de violação $(5,0 \%)$ e a violência sexual $(2,7 \%)$ tiveram uma frequência 
relativamente baixa de denúncias ao longo dos anos. Vale destacar que ao longo dos anos a frequência das denúncias não variou muito em relação aos diferentes tipos de violações.

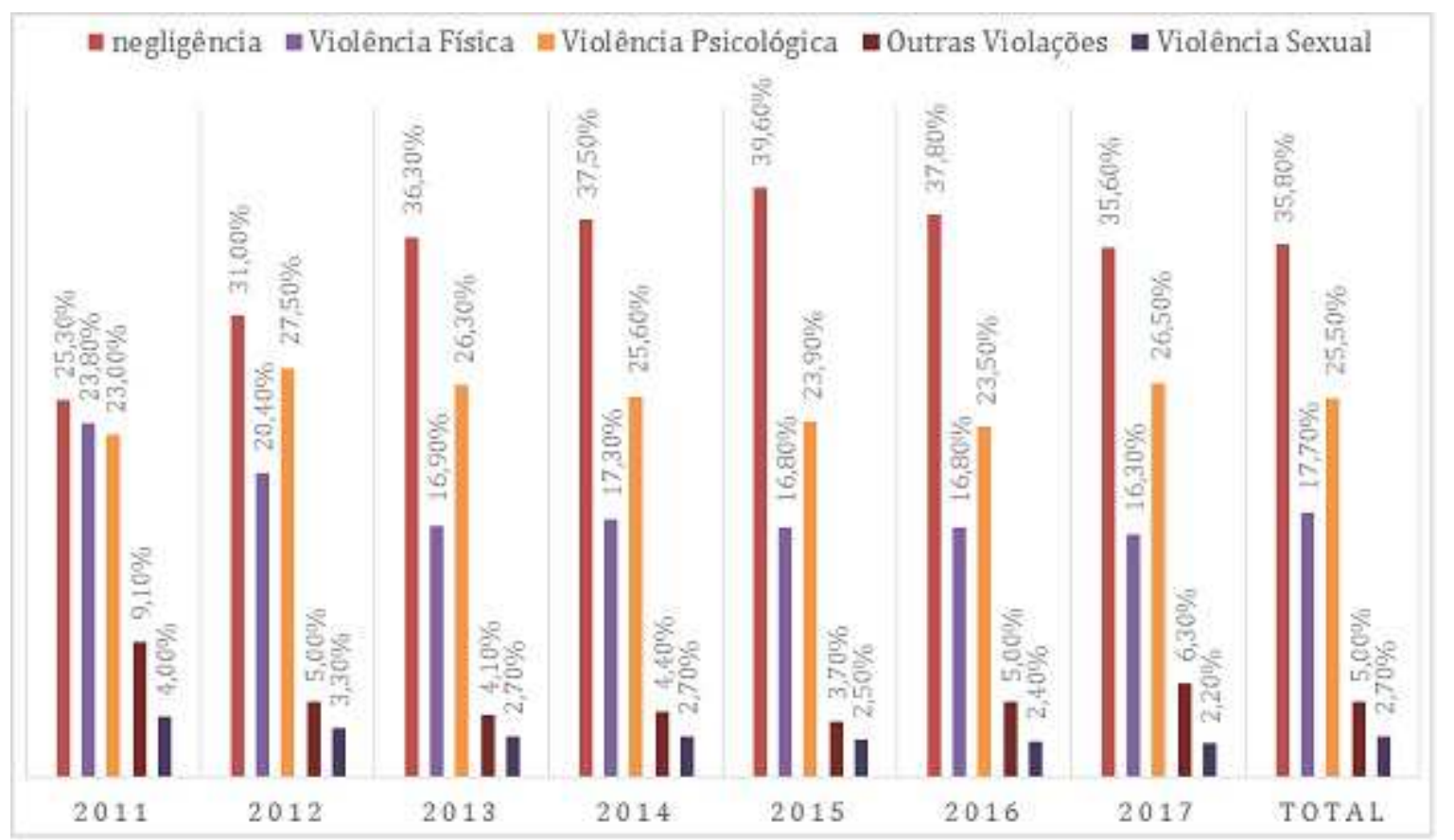

Figura 2. Tipos de violação contra pessoas com deficiência denunciadas no Brasil no período de 2011 a 2017.

\section{Perfil das Vítimas com Deficiência}

Conforme os dados do balanço geral do Disque 100, a maior parte das vítimas de 2011 a 2017 são jovens com idade entre 18 e 30 anos (29\%), seguidas de adultos entre 31 e 40 anos (24\%), 41 a 50 anos $(23,9 \%)$ e 51 a 60 anos (20,2\%). Desconsiderando-se o número de casos em que o sexo das vítimas não foi informado, o sexo feminino lidera as denúncias do período, totalizando 52,8\% dos casos, enquanto o sexo masculino representa 47,2\% das denúncias. Quanto à identidade de gênero ${ }^{3}$, em $94,6 \%$ das denúncias não foi informada, $5 \%$ foram descritas como pessoas com deficiência heterossexuais, e $0,4 \%$ restantes dessa população, se dividem entre bissexuais, gays, lésbicas, transexuais e travestis.

Em relação à etnia das vítimas, na maioria das denúncias ela não foi informada $(\mathrm{n}=22.232 ; 33,63 \%)$. Nas denúncias onde consta essa informação, a maior parcela refere-se a pessoas pardas $(29,8 \%)$, seguido por brancas $(28,1 \%)$, pretas $(7,8 \%)$, amarelas $(0,5 \%)$ e indígenas $(0,2 \%)$. Quanto ao tipo de deficiência das vítimas, a maioria tinha deficiência 
intelectual $(65,8 \%)$, seguida das deficiências física $(22,1 \%)$, visual $(4,9 \%)$ e auditiva $(3 \%)$. O total não informado foi de $4,2 \%$.

\section{Perfil dos Suspeitos}

De acordo com dados do Disque 100, desconsiderado o número de denúncias onde não foi informada a idade dos suspeitos, a faixa etária entre 18 a 30 anos foi a mais frequente nas denúncias (24,6\%), seguida daqueles entre 41 e 50 anos (22,8\%), e de 31 a 40 anos (22,7\%). Porém é necessário considerar que o percentual de casos onde a idade do suspeito não foi informada supera esses números $(43,7 \%)$. Quanto ao sexo dos suspeitos, desconsiderando os casos onde não há informação, a maioria dos suspeitos $(57,6 \%)$ foi identificada como sendo do sexo masculino e $42,4 \%$ do sexo feminino. Em relação à etnia dos suspeitos, nos casos onde havia essa informação, $43,7 \%$ foi descrito como branco, $42,7 \%$ pardo, $12,5 \%$ preto, $0,7 \%$ amarelo e $0,3 \%$ indígena.

\section{Relações entre Suspeitos e Vítimas}

As relações entre suspeitos e vítimas informadas nas denúncias, evidenciam que a maioria dos suspeitos eram pessoas da convivência da vítima (ver Tabela 1). O maior índice de suspeitos encontrava-se entre pessoas da família nuclear $(43,6 \%)$, destacando-se entre estes, os irmãos (19,8\%). Em seguida destacam-se os vizinhos e os cuidadores. 
Tabela 1

Relação entre Suspeito e Vitima

\begin{tabular}{|c|c|c|c|c|c|}
\hline \multicolumn{3}{|c|}{ FAMILIA NUCLEAR } & \multicolumn{3}{|c|}{ PARCEIROS AFETIVOS } \\
\hline & $\mathrm{n}$ & $\%$ & & $\mathrm{n}$ & $\%$ \\
\hline Immão(ã) & 19.857 & 20 & Marido & 2.392 & 2,4 \\
\hline Filho(a) & 10.448 & 10 & Esposa & 1.249 & 1,2 \\
\hline Mãe & 9,085 & 9 & Companheiro(a) & 1.239 & 1,2 \\
\hline Pai & 4.347 & 4 & Ex companheiro(a) & 683 & 0,7 \\
\hline \multirow[t]{3}{*}{ Total } & 43.737 & 44 & Ex marido & 512 & 0,5 \\
\hline & A ESTEN & & Ex esposa & 288 & 0,3 \\
\hline & $\mathrm{n}$ & $\%$ & Namorado(a) & 156 & 0,2 \\
\hline Cunhado(a) & 2.691 & 2,69 & Total & 6.519 & 6,5 \\
\hline Sobrinho(a) & 2.510 & 2,50 & \multicolumn{3}{|c|}{ OUTROS VINCULOS } \\
\hline Tio(a) & 2.142 & 2,14 & & $\mathrm{n}$ & $\%$ \\
\hline Familiares & 1.534 & 1,53 & Vizinho & 5.631 & 5,62 \\
\hline Padrasto & 1.151 & 1,15 & Cuidador & 1.170 & 1,17 \\
\hline Primo & 1.065 & 1,06 & Amigo & 443 & 0,44 \\
\hline Genro/Nora & 937 & 0,94 & Empregador & 398 & 0,40 \\
\hline Madrasta & 539 & 0,54 & Diretor(a) de escola & 225 & 0,22 \\
\hline Avós & 339 & 0,34 & Empregado(a) & 201 & 0,20 \\
\hline Sogro(a) & 244 & 0,24 & Professor(a) & 195 & 0,19 \\
\hline Enteado(a) & 222 & 0,22 & Lider religioso & 64 & 0,06 \\
\hline Neto(a) & 202 & 0,20 & $\begin{array}{l}\text { Diretor(a) de unidade } \\
\text { prisional }\end{array}$ & 60 & 0,06 \\
\hline Padrinho/Madrinha & 15 & 0,02 & Subordinado(a) & 52 & 0,05 \\
\hline Bisneto(a) & 3 & 0,00 & Total & 8.439 & 8,42 \\
\hline Total & 13.594 & 13,56 & & & \\
\hline Não informado & 19.465 & 19,4 & & & \\
\hline Desconhecido & 8.406 & 8,38 & & & \\
\hline Própria vitima & 98 & 0,09 & & & \\
\hline TOTAL & 100.258 & 100 & & & \\
\hline
\end{tabular}

\section{Local Onde Ocorreu a Violação}

Conforme os dados analisados, a maioria dos episódios de violência ocorreu na casa da própria vítima $(64,8 \%)$, seguido da casa do suspeito $(12,1 \%)$, em uma casa não especificada (9\%), na rua $(7,2 \%)$ e em outros locais $(6,9 \%)$.

\section{Discussão}

Ao longo da história da humanidade são vários os relatos de violência contra pessoas com deficiência (Pessotti, 1984), no entanto, ainda há carência de estudos que abordem essa temática tanto nacional quanto internacionalmente. Por exemplo, em revisões sistemáticas de literatura e meta-analises a respeito da prevalência dos casos de violência de adultos (Hughes et al., 2012) e crianças e adolescentes (Jones et al., 2012), os autores destacaram as lacunas e limitações nos estudos existentes em algumas regiões do mundo, particularmente em países em desenvolvimento. Considerando o objetivo de analisar os dados oficiais do Disque 100 em 
relação à violência contra pessoas com deficiência de modo a caracterizar esse fenômeno no Brasil buscamos contribuir para diminuir essa lacuna no país.

Embora a atenção a esse tema no Brasil seja relativamente recente, visto que os dados em órgãos oficiais só passaram a ser computados a partir de 2011, houve um número considerável de denúncias ao longo dos anos $(M=8800$, dp=2879). Analisando- se a Figura 1, após a implantação do serviço, houve uma curva acelerada de denúncias ao longo dos anos, atingindo certa estabilidade (na faixa de 8 a 10 mil denúncias/ano). Vale destacar que as tentativas de caracterizar a violência contra pessoas com deficiência comumente partem da perspectiva de dados subestimados, seja devido à noção que um número considerável de violações não é identificado e/ou denunciado, seja pela probabilidade de impunidade dos agressores, pelo medo de denunciar, pela falta de conhecimento de leis de proteção ou mesmo pelas crenças equivocadas de inferioridade e desvalorização da pessoa com deficiência.

No tocante ao tipo de violência mais praticado contra pessoas com deficiência, os dados indicaram a negligência como sendo a mis frequente $(35,8 \%$ das denúncias ao longo dos anos). Entre os tipos de negligência mais denunciados, destaca-se a negligência em amparo e responsabilização, dando indícios de que, nesses casos, as pessoas com deficiência foram lançadas à própria sorte. Sugere-se que tal dado seja reflexo da falta de informação e apoio dos familiares/cuidadores, além das exigências de cuidado e estresse dos cuidadores (UNICEF, 2013). Em decorrência disso, reitera-se a importância de oferecer redes de apoio e informações aos familiares e responsáveis pela pessoa com deficiência.

Pesquisas realizadas com famílias de crianças com deficiência relatam que as mesmas necessitam de uma rede de apoio social para manter o equilíbrio e a harmonia (Gualda, Borges, \& Rodrigues, 2013), podendo esta ser composta por parentes, amigos, serviços de educação, saúde e assistência, religioso, dentre outros (Maia \& Williams, 2005). Essa rede pode tanto oferecer apoio emocional e instrumental/material, quanto fornecer informações adequadas sobre como lidar com filhos com deficiência, visto que algumas famílias não sabem como lidar com as limitações de seus filhos, e não têm recursos ou suporte para promover os cuidados necessários e adequados à pessoa com deficiência.

A violência psicológica, que consta como a segunda forma de violência mais frequente, tendo o maior número de denúncias referentes à hostilização e a humilhação da pessoa com deficiência. Fatores que justificam esse tipo de violência contra pessoas com deficiência podem ser o estigma associado à deficiência, a desvalorização desses indivíduos em nossa sociedade e a invisibilidade dos mesmos. Pessoas com deficiência são, com frequência, consideradas pela sociedade como sendo não completamente humanas, e tendo 
menor valor (Ferreira, 2008). Desse modo, destaca-se a importância das políticas de inclusão, como forma de trazer essas pessoas para o convívio social, diminuindo, assim, o estigma e o preconceito existentes, fazendo com que elas, ao serem participativas no organismo social, passem a ser respeitadas como sujeitos de direitos.

Denunciar a violência e contribuir socialmente para a sua erradicação é condição do processo de democratização. A Lei Brasileira de Inclusão da Pessoa com Deficiência (Lei n. 13.146, 2015), em seu artigo $7^{\circ}$, define que é "dever de todos comunicar à autoridade competente qualquer forma de ameaça ou de violação aos direitos da pessoa com deficiência". Todo cidadão tem o dever legal de denunciar qualquer forma de violação de direitos. Assim, quando estamos diante de uma atitude de desrespeito, preconceito e de violência contra uma pessoa com deficiência, não podemos nos omitir e devemos procurar autoridades competentes para efetivação da defesa desses direitos.

Quanto a características da vítima, nota-se que as vítimas eram em sua maioria mulheres $(52,8 \%)$, pardas $(29,7 \%)$, com idade entre 18 a 50 anos $(76,9 \%)$ e com deficiência intelectual $(65,8 \%)$. Tais dados vão ao encontro de pesquisas que indicam que gênero e tipo de deficiência são fatores de risco importantes (UNICEF, 2013). Por exemplo, no relatório sobre a situação mundial da infância, realizado pelo Fundo das Nações Unidas para a Infância (UNICEF), notou-se que crianças com deficiência intelectual são mais vulneráveis a serem vítimas de abuso sexual do que seus pares - probabilidade 4,6 vezes mais alta (UNICEF, 2013), o que dá certo suporte empírico à teoria de que indivíduos com deficiência intelectual são vistos como mais desamparados e, consequentemente, vítimas ideais para alguns abusadores (Petersilia, 2001).

Quanto à idade das vítimas, a maioria entre 18 e 50 anos (69,2 \%), sugere-se que a vulnerabilidade estaria associada à expectativa social quanto à independência e autonomia do indivíduo, isto é, é esperado que indivíduos nessa fase do desenvolvimento tenham capacidade de fazer suas escolhas, consigam realizar as atividades relacionadas aos autocuidados/alimentação sozinho e, preferencialmente, tenha independência financeira (Papalia, Olds, \& Feldman, 2006). Contudo, a pessoa com deficiência pode continuar a demandar cuidados nessa fase do desenvolvimento, gerando, consequentemente, maiores conflitos.

De acordo com os dados, os suspeitos em sua grande maioria são pessoas das relações dos indivíduos com deficiência $(72,1 \%)$, branco, do sexo masculino $(57,6 \%)$, com idade entre 18 e 50 anos (39,6\%), embora em um número considerável de denúncias (43,7\%) esse dado não foi informado. Cumpre destacar que na maioria dos casos $(57,1 \%)$ o suspeito de cometer 
violência contra a pessoa com deficiência era um membro da família, levantando a necessidade dos profissionais que trabalham com essa população serem treinados a identificar e denunciar casos suspeitos de abuso (Hibbard \& Desch, 2007; Manders \& Stoneman, 2009).

Com relação ao grau de parentesco entre vítima e agressor, os irmãos apareceram em primeiro lugar no número de suspeitos, chegando a quase $20 \%$ das denúncias. Tal dado suscita a necessidade de discutir as implicações que surgem do fato de se ter irmãos com deficiência. Esses irmãos, muitas vezes, assumem responsabilidades no cuidado dos irmãos com deficiência e da casa desde muito cedo (Messa \& Fiamenghi, 2010; Almeida \& Silva, 2015; Burke, 2010; Meyers \& Vipond, 2005; Stoneman, 2005; Soares, Franco, \& Carvalho, 2009; Trent, Kaiser, \& Wolery, 2005); além disso, muitos relatam sentimentos de solidão e ressentimento por se sentirem negligenciados por pais e profissionais que trabalham com a família, devido ao foco no irmão com deficiência, somado ao sentimento de raiva e ciúmes por perceberem que os irmãos estão sendo favorecidos com maior atenção e presentes (Messa \& Fiamenghi, 2010). Ademais, dados de pesquisas realizadas com irmãos de pessoas com deficiência indicam que estes comumente têm pouco conhecimento a respeito da deficiência do irmão (Matsukura \& Cid, 2008; Messa \& Fiamenghi Jr., 2010; Nunes, Silva, \& Aiello, 2008; Stoneman, 2005; Petean \& Suguihura, 2005), o que pode acarretar em prejuízos na convivência fraternal e na condição emocional do irmão com desenvolvimento típico.

\section{Considerações Finais}

A caracterização da violência contra pessoas com deficiência no Brasil revelou, principalmente, uma grave situação de violência intrafamiliar/doméstica, pois aqueles que deveriam zelar pela segurança das pessoas com deficiência configuram como a maioria entre os suspeitos nas denúncias ao Disque 100. Destaca-se a vulnerabilidade de mulheres com deficiência à violência no ambiente doméstico, o que nos permite considerar que, para além da deficiência, o gênero também é uma variável importante a ser considerada. Apesar dos dados analisados permitirem desvendar um fenômeno pouco explorado em pesquisas nacionais e internacionais, a presente pesquisa apresenta algumas limitações, tais como a dificuldade em acessar o protocolo utilizado pela equipe do Disque 100, de modo a compreender o teor das perguntas realizadas, além do número relativamente alto de dados não informados.

O resultado da análise dos dados sinaliza a necessidade de ações de prevenção à violência contra pessoas com deficiência através de redes de apoio, que possam contribuir na 
promoção do equilíbrio das relações familiares, dando suporte às pessoas com deficiência, assim como para sua família. Também são necessárias mudanças relativas à visão da pessoa com deficiência na sociedade, além da garantia de acesso a serviços.

É necessário ressaltar que o país tem buscado mecanismos de inclusão e, consequentemente, de proteção dos direitos e da dignidade das pessoas com deficiência, fato que pode ser observado na evolução do serviço Disque 100. Analisando o histórico do serviço, é possível notar o reflexo das políticas de inclusão e proteção das pessoas com deficiência, tanto no número de denúncias, quanto nos tipos de violações denunciadas. A Lei Brasileira de Inclusão da Pessoa com Deficiência (Lei n. 13.146, 2015) pode ser considerada um importante marco jurídico na efetiva promoção da igualdade, acessibilidade e respeito aos direitos da pessoa com deficiência. A responsabilidade por seu cumprimento recai sobre entes públicos e a sociedade civil, que deverão envidar esforços conjuntos para concretização da efetiva inclusão desses sujeitos de direito.

\section{Referências}

Abramovay, M., Castro, M. G., Pinheiro, L. C., Lima, F. S., \& Martinelli, C. C. (2002). Juventude, violência e vulnerabilidade social na América Latina: Desafios para políticas públicas. Brasília: UNESCO, BID.

Almeida, B. R., \& Pereira-Silva, N. L. (2015). A convivência com um irmão com síndrome de Down. Psicologia em Pesquisa, 9(1), 10-19. doi:10.5327/Z1982-1247201500010003

Andrade, V. N. G. (2017). Transfobia no percurso denunciativo brasileiro: Um estudo a partir do Disque Direitos Humanos da Presidência da República (Tese de Doutorado). Pontifícia Universidade Católica de Goiás, Goiânia, Brasil. Recuperado de http://tede2.pucgoias.edu.br:8080/handle/tede/3949

Andrade, V. N. G. (2012). As falas dos atendentes do Disque 100 sobre a escuta das denúncias de abuso sexual contra crianças e adolescentes (Dissertação de Mestrado). Pontifícia Universidade Católica de Goiás, Goiânia, Brasil. Recuperado de http://tede2.pucgoias.edu.br:8080/handle/tede/1804

Assis, S. G., Avanci, J. Q., Pesce, R. P., \& Ximenes, L. F. (2009). Situação de crianças e adolescentes brasileiros em relação à saúde mental e à violência. Ciência \& Saúde Coletiva, 14(2), 349-361. doi:10.1590/S1413-81232009000200002 
Barros, A. C. M. W., Deslandes, S. F., \& Bastos, O. M. (2016). A violência familiar e a criança e o adolescente com deficiências. Cadernos de Saúde Pública, 32(6), 1-7. doi:10.1590/0102-311X00090415

Bernardes, L. F., \& Moreira, M. I. C. (2013) Metodologia de intervenção do disque 100: Perspectivas e desafios. In S. M. G. Souza, M. I. C. Moreira (Orgs.), Quebrando o silêncio: Disque 100 estudo sobre a denúncia de violência sexual contra crianças e adolescentes no Brasil (pp. 149-159). Goiânia: Cânone.

Brasil. (2012). Convenção sobre os Direitos das Pessoas com Deficiência (2007): Convenção sobre os Direitos das Pessoas com Deficiência: Protocolo Facultativo à Convenção sobre os Direitos das Pessoas com Deficiência: Decreto legislativo $n^{o}$ 186, de 09 de julho de 2008: Decreto $n^{o}$ 6.949, de 25 de agosto de 2009. Brasília: Secretaria de Direitos Humanos, Secretaria Nacional de Promoção dos Direitos da Pessoa com Deficiência. Recuperado de http://www.crianca.mppr.mp.br/arquivos/File/publi/sedh/convencaopessoascomdeficie ncia_2012.pdf

Brasil (2015). Lei n. 13.146, de 6 de Julho de 2015. Institui a Lei Brasileira de Inclusão da Pessoa com Deficiência (Estatuto da Pessoa com Deficiência). Brasília, DF: Presidência da República. Recuperado de http://www.planalto.gov.br/ccivil_03/_ato2015-2018/2015/lei/113146.htm

Burke, P. (2010). Brother and sisters of disabled children: The experience of disability by association. British Journal of Social Work, 40(6), 1681-1699. doi:10.1093/bjsw/bcp088

Cavalcante, F. G., Marinho, A. S. N., Bastos, O. M., Deus, V. V., Maimone, M. S., Carvalho, M. M., Fiaux, M. P., \& Valdene, R. S. R. (2009). Diagnóstico situacional da violência contra crianças e adolescentes com deficiência em três instituições do Rio de Janeiro. Ciência \& Saúde Coletiva, 14(1), 45-56. doi:10.1590/S1413-81232009000100010

Coordenadoria Nacional para Integração da Pessoa Portadora de Deficiência. (2008). A Convenção sobre Direitos das Pessoas com Deficiência Comentada. Brasília, DF: Secretaria Especial dos Direitos Humanos da Presidência da República.

Costa, N. G. (2016). Do disque denúncia ao call center: Os limites do Disque 100 para a realização da denúncia de violência contra a população LGBT (Dissertação de Mestrado). Universidade Federal de Minas Gerais, Belo Horizonte, Brasil. Recuperado de http://www.fafich.ufmg.br/pospsicologia/egressos/dissertacoesmestrado/dissertacoes-mestrado-defendidas-em-2016/ 
Cruz, D. M. C., Silva, J. T., \& Alves, H. C. (2007). Evidências sobre violência e deficiência: Implicações para futuras pesquisas. Revista Brasileira de Educação Especial, 13(1), 131-146. doi:10.1590/S1413-65382007000100009

Day, V. P., Telles, L. E. B., Zoratto, P. H., Azambuja, M. R. F., Machado, D. A., Silveira, M. B., Debiaggi, M., Reis, M. G., Cardoso, R. G., \& Blank, P. (2003). Violência doméstica e suas diferentes manifestações. Revista de Psiquiatria do Rio Grande do Sul, 25(suppl. 1), 9-21. doi:10.1590/S0101-81082003000400003

Faraj, S. P., Siqueira, A. C., \& Arpini, D. M. (2016). Notificação da Violência: Percepções de Operadores do Direito e Conselheiros Tutelares. Psicologia: Ciência e Profissão, 36(4), 907-920. doi:10.1590/1982-3703000622014.

Ferreira, W. B. (2008). Vulnerabilidade à Violência Sexual no Contexto da Escola Inclusiva: Reflexão Sobre a Invisibilidade da Pessoa com Deficiência. Revista Electrónica Iberoamericana sobre Calidad, Eficácia y Cambio em Educcion. 6(2), 120-136. Recuperado de http://hdl.handle.net/10486/661104

Gualda, D. S., Borges, L., \& Rodrigues, R. K. G. (2013). A participação da família de crianças pré-escolares público alvo da educação especial no processo de Escolarização dos filhos. In F. E. Denari (Org.). Educação especial: Reflexos sobre o dizer e o fazer (pp. 79-100). São Carlos: Pedro e João Editores.

Gualda, D., Borges, L., \& Cia, F. (2013). Famílias de crianças com necessidades educacionais especiais: recursos e necessidades de apoio. Revista Educação Especial, 26(46), 307330. doi:10.5902/1984686X5379

Hibbard, R. A., \& Desch, L. W. (2007). Maltreatment of children with disabilities. Pediatrics, 119(5), 1018-1025. doi:10.1542/peds.2007-0565

Hughes, K., Bellis, M. A, Jones L., Wood S., Bates G., Eckley L., McCoy E., Mikton C., Shakespeare T., \& Officer A. (2012). Prevalence and risk of violence against adults with disabilities: A systematic review and meta-analysis of observational studies. Lancet, 379(9826), 1621-1629, doi:10.1016/S0140-6736(11)61851-5

Jones L., Bellis M. A., Wood S., Hughes K., McCoy E., Eckley L., Bates G., Mikton C., Shakespeare T., \& Officer A. (2012). Prevalence and risk of violence against children with disabilities: A systematic review and meta-analysis of observational studies. Lancet, 380(9845), 899-907, doi:10.1016/S0140-6736(12)60692-8

Maia, J. M. D., \& Williams, L. C. A. (2005). Fatores de risco e fatores de proteção ao desenvolvimento infantil: Uma revisão da área. Temas em Psicologia, 13(2), 91-103. 
Recuperado de http://pepsic.bvsalud.org/scielo.php?script=sci_arttext\&pid=S1413389X2005000200002

Maior, I. M. M. L. (2008). Apresentação. In A. P. C. Rezende \& F. M. P. Vital (Coords.), A Convenção sobre Direitos das Pessoas com Deficiência Comentada (pp. 20-22). Brasília: Secretaria Especial dos Direitos Humanos, Coordenadoria Nacional para Integração da Pessoa Portadora de Deficiência.

Manders, J. E., \& Stoneman, Z. (2009). Children with disabilities in the child protective services system: An analog study of investigation and case management. Child Abuse \& Neglect, 33(4), 229-237. doi:10.1016/j.chiabu.2008.10.001

Masuchi, M., \& Rocha, E. (2012). Cuidar de pessoas com deficiência: Um estudo junto a cuidadores assistidos pela estratégia da saúde da família. Revista De Terapia Ocupacional Da Universidade De São Paulo, 23(1), 89-97. doi:10.11606/issn.22386149.v23i1p89-97

Matsukura, T. S., \& Cid, M. F. B. (2008). Irmãos de crianças com necessidades especiais e suas famílias: Diferentes expressões sobre essa realidade. Cadernos de Terapia Ocupacional UFSCar, 16(1), 7-16. Recuperado de http://www.cadernosdeterapiaocupacional.ufscar.br/index.php/cadernos/article/view/1 29

Messa, A. A., \& Fiamenghi Jr., G. A. (2010). O impacto da deficiência nos irmãos: Histórias de vida. Ciência \& Saúde Coletiva, 15(2), 529-538. doi:10.1590/S141381232010000200029

Messeder, S. A., Boaventura, L. S., \& Oliveira Junior, W. A. (2017). Um estudo sobre os tipos de denúncias de violência registradas no disque 100 correlata às pessoas lgbt no âmbito do estado baiano. Seminário internacional: Enlaçando sexualidades: 10 anos, Salvador, Bahia, Brasil, 5. Recuperado de https://www.editorarealize.com.br/revistas/enlacando/trabalhos/TRABALHO_EV072_ MD1_SA13_ID741_17072017160915.pdf

Meyers, C., \& Vipond, J. (2005). Play and social interactions between children with developmental disabilities and their siblings: A systematic literature review. Physical \& Occupational Therapy in Pediatrics, 25(1-2), 81-103. doi:10.1080/J006v25n01_06

Minayo, M. C. S. (2014). Manual de enfrentamento à violência contra a pessoa idosa: É possível prevenir: É necessário superar. Brasília, DF: Secretaria de Direitos Humanos da Presidência da República. 
Ministério dos Direitos Humanos. (2018). Disque 100- Balanço Geral 2011 a 2017: Pessoa com deficiência. Brasília, DF: Autor. Recuperado de http://www.mdh.gov.br/informacao-ao-cidadao/ouvidoria/balanco-disque-100

Montibeller, B. (2012). Os Direitos Humanos no Brasil e a Constituição de 1988: O desafio da efetivação dos Direitos Humanos. Brasília: Portal do governo, inclusão digital e sociedade do conhecimento. Recuperado de http://www.planejamento.gov.br/assuntos/logistica-e-tecnologia-dainformacao/noticias/portal-de-inclusao-digital/

Moreira, M. C. N., Bastos, O. M., Bastos, L. C., Soares, A. H. R., Souza, W. S., \& Sanchez, R. N. (2014). Violência contra crianças e adolescentes com deficiência: Narrativas com conselheiros tutelares. Ciência \& Saúde Coletiva, 19(9), 3869-3878. doi:10.1590/1413-81232014199.12172013

Nunes, C. C., \& Aiello, A. L. R. (2004). O convívio com irmão especial e a caracterização da interação: Um estudo descritivo. Revista Brasileira de Educação Especial, 10(2), 143 160. Recuperado de https://abpee.net/revista-brasileira-de-educacao-especial-vol-10no- $2 /$

Nunes, C. C., Silva, N. C. B., \& Aiello, A. L. R. (2008). As contribuições do papel do pai e do irmão do indivíduo com necessidades especiais na visão sistêmica da família. Psicologia: Teoria e Pesquisa, 24(1), 37-44. doi:10.1590/S0102-37722008000100005

Papalia, D. E., Olds, S. W., \& Feldman, R. D. (2006). Desenvolvimento Humano (10a ed., C. F. Marques, Trad.) Porto Alegre: AMGH.

Pereira, B. M. S., Lopes, L. C., \& Gomes, T. C. (2015). Violência contra Pessoas com Deficiência: É quase invisível, mas existe. São Paulo: SEDPCD. Recuperado de http://violenciaedeficiencia.sedpcd.sp.gov.br/pdf/textosApoio/Texto13.pdf

Pessoti, I. (1984). Deficiência mental: Da superstição à ciência. São Paulo: TAQ EDUSP.

Petean, E. B. L., \& Suguihura, A. L. M. (2005). Ter um irmão especial: Convivendo com a Síndrome de Down. Revista Brasileira de Educação Especial, 11(3), 445-460. doi:10.1590/S1413-65382005000300009

Petersilia, J. R. (2001). Crime victims with developmental disabilities: A review essay. Physical \& Occupational Therapy in Pediatrics, 28(6), 81-103. doi:10.1177/009385480102800601

Silva, M. C. M., Brito, A. M., Araújo, A. L., \& Abath, M. B. (2013). Caracterização dos casos de violência física, psicológica, sexual e negligências notificados em Recife, 
Pernambuco, 2012. Epidemiologia e Serviços de Saúde, 22(3), 403-412. doi:10.5123/S1679-49742013000300005

Soares, M. P. G., Franco, A. L. S., \& Carvalho, A. M. A. (2009). Crianças que cuidam de irmãos com necessidades especiais. Psicologia: Teoria e Pesquisa, 25(1), 45-54. doi:10.1590/S0102-37722009000100006

Stoneman, Z. (2005). Siblings of children with disabilities: Research themes. Mental Retardation, 43(5), 339-350. doi:10.1352/00476765(2005)43[339:SOCWDR]2.0.CO;2

Tavares, R. C., Louredo, L. C. R. S., \& Prado, J. I. A. (2013). Breve história do disque 100: Análise da implantação e monitoramento do serviço. In S. M. G. Souza, M. I. C. Moreira (Orgs.), Quebrando o silêncio: disque 100: estudo sobre a denúncia de violência sexual contra crianças e adolescentes no Brasil (pp. 113-147). Goiânia: Cânone.

Trent, J. A., Kaiser, A. P., \& Wolery, M. (2005). The use of responsive interaction strategies by siblings. Topics in Early Childhood Special Education, 25(2), 107-118. doi:10.1177/02711214050250020101

UNICEF (2013). Situação mundial da infância: Crianças com deficiência. New York, NY: Autor. Recuperado de https://www.unicef.pt/docs/PT_SOWC2013.pdf

Williams, L. C. A. (2003). Sobre deficiência e violência: Reflexões para uma análise de revisão de área. Revista Brasileira de Educação Especial, 9(2), 141-154. Recuperado de http://www.laprev.ufscar.br/documentos/arquivos/artigos/2003-williams.pdf

\section{Endereço para correspondência}

Márcia de Lima

Rua Anita Stella, 270, Vila Celina, São Carlos - SP, Brasil. CEP 13566-447

Endereço eletrônico: helemarcia@gmail.com

\section{Sabrina Mazo D'Affonseca}

Rua Quintino Bocaiuva, 524, Bela Vista, São Carlos - SP, Brasil. CEP 13574-003

Endereço eletrônico: samazo@ @otmail.com

Recebido em: 29/05/2019

Reformulado em: 03/04/2020

Aceito em: 08/05/2020 


\section{Notas}

* Educadora especial pela Universidade Federal de São Carlos.

** Professora Adjunta do Departamento de Psicologia e do Programa de Pós Graduação em Psicologia da Universidade Federal de São Carlos; Laboratório de Análise e Prevenção da Violência-Laprev.

${ }^{1}$ Disponível em: https://www.mdh.gov.br/informacao-ao-cidadao/disque-100-1

${ }^{2}$ Disponível em: https://www.mdh.gov.br/informacao-ao-cidadao/ouvidoria/balanco-disque-100

${ }^{3}$ É necessário destacar que os dados apresentados no documento consultado não diferenciam identidade de gênero e orientação sexual. Entretanto, acompanhando as discussões atuais no campo dos estudos de gênero e sexualidade, a identidade de gênero refere-se ao gênero com que a pessoa se identifica (masculino/feminino/não binário), podendo ou não concordar com o gênero que lhe foi atribuído ao nascimento. Ademais, identidade de gênero e orientação sexual (heterossexual, homossexual, bissexual e assexual) são dimensões diferentes. Nesse sentido, a categorização dos dados no documento consultado é imprecisa.

Este artigo de revista Estudos e Pesquisas em Psicologia é licenciado sob uma Licença Creative Commons Atribuição-Não Comercial 3.0 Não Adaptada. 